\title{
Degree Square Sum Polynomial of some Special Graphs
}

\author{
B. Basavanagoud* and Chitra $\mathbf{E}^{1}$ \\ Department of Mathematics, Karnatak University, Dharwad - 580003 \\ Karnataka, India.
}

\begin{abstract}
Degree square sum matrix $D S S(G)$ of a graph $G$ is a square matrix of order equal to the order of $G$ with its $(i, j)^{t h}$ entry as $d_{i}^{2}+d_{j}{ }^{2}$ if $i \neq j$ and zero otherwise, where $d_{i}$ and $d_{j}$ are the degrees of $i^{\text {th }}$ and $j^{\text {th }}$ vertices of $G$ respectively. In this paper, we obtain the degree square sum polynomial of some special graphs.
\end{abstract}

AMS subject classification: $05 \mathrm{C} 50$.

Keywords: Degree square sum matrix, degree square sum polynomial, transformation graph, graph operation.

\section{INTRODUCTION}

We consider in this paper, a graph $G$ as a simple, undirected graph with $n$ vertices and $m$ edges. Let $V(G)$ be a vertex set and $E(G)$ be an edge set of $G$. The degree $d_{G}(v)$ of a vertex $v \in V(G)$ is the number of edges incident to it in $G$. The graph $G$ is $r$-regular if the degree of each vertex in $G$ is $r$. Let $v_{1}, v_{2}, \ldots, v_{n}$ be the vertices of $G$ and let $d_{i}=d_{G}\left(v_{i}\right)$. For undefined graph theoretic terminologies and notations, we refer [11] or [15].

The literature of Graph Theory has several graph polynomials based on the matrices associated with a graph. In [4], We have introduced degree square sum matrix wherein we have given bounds for degree square sum energy of a graph and we have computed the degree square sum polynomial of graphs obtained by some graph operations. In this paper, we obtain the degree square sum polynomial of some special graphs.

The degree square sum matrix of a graph $G$ is an $n \times n$ matrix denoted by $\operatorname{DSS}(G)=\left[d s s_{i j}\right]$ and whose elements are defined as

$$
d s s_{i j}= \begin{cases}d_{i}^{2}+d_{j}^{2} & \text { if } i \neq j, \\ 0 & \text { otherwise. }\end{cases}
$$

Let $I_{n}$ be an identity matrix (order $\mathrm{n}$ ) and $J_{n}$ be a matrix (order $\mathrm{n}$ ) whose all entries are equal to 1 . The degree square sum polynomial of a graph $G$ is defined as

$$
P_{D S S(G)}(\mu)=\operatorname{det}\left(\mu I_{n}-D S S(G)\right) .
$$

Let $\mu_{1}, \mu_{2}, \ldots, \mu_{n}$ are called the degree square sum eigenvalues of $G$. For an $r$-regular graph of order $n$,

$$
D S S(G)=2 r^{2} J_{n}-2 r^{2} I_{n}
$$

and

$$
P_{D S S(G)}(\mu)=\left[\mu-2 r^{2}(n-1)\right]\left[\mu+2 r^{2}\right]^{n-1} .
$$

For more details on degree square sum polynomial of a graph, refer [4].

Lemma 1. [20] If $a, b, c$ and $d$ are real numbers, then the determinant of the form

$$
\left|\begin{array}{cc}
(\mu+a) I_{n_{1}}-a J_{n_{1}} & -c J_{n_{1} \times n_{2}} \\
-d J_{n_{2} \times n_{1}} & (\mu+b) I_{n_{2}}-b J_{n_{2}}
\end{array}\right|
$$

of order $n_{1}+n_{2}$ can be expressed in the simplified form as $(\mu+a)^{n_{1}-1}(\mu+b)^{n_{2}-1}\left\{\left[\mu-\left(n_{1}-1\right) a\right]\left[\mu-\left(n_{2}-1\right) b\right]-n_{1} n_{2} c d\right\}$.

The above lemma is useful for proving the forthcoming theorems. 


\section{DEGREE SQUARE SUM POLYNOMIAL OF SOME CYCLE RELATED AND PRODUCT RELATED GRAPHS}

The details of the following graphs can be found in [10].

Let $C_{n}^{(t)}$ denote the one-point union of $t$ cycles of length $n$. The graph $C_{3}^{(t)}$ is called a Friendship graph.

Theorem 2. If $C_{3}^{(t)}$ is a friendship graph of order $2 t+1$, then the degree square sum polynomial of $C_{3}^{(t)}$ is

$$
P_{D S S\left(C_{3}^{(t)}\right)}(\mu)=(\mu+8)^{n-2}\left\{[\mu-8(n-2)]-(n-1)\left[(n-1)^{2}+4\right]^{2}\right\} .
$$

Proof. The graph $C_{3}^{(t)}$ of order $2 t+1$ has two types of verices namely $n-1$ vertices of degree 2 and 1 vertices of degree $n-1$. Hence,

$$
\operatorname{DSS}\left(C_{3}^{(t)}\right)=\left[\begin{array}{cc}
8\left(J_{n-1}-I_{n-1}\right) & \left(2^{2}+(n-1)^{2}\right) J_{(n-1) \times 1} \\
\left(2^{2}+(n-1)^{2}\right) J_{1 \times(n-1)} & 2(n-1)^{2}\left(J_{1}-I_{1}\right)
\end{array}\right]
$$

Therefore,

$$
\begin{aligned}
P_{D S S\left(C_{3}^{(t)}\right)}(\mu) & =\left|\mu I-\operatorname{DSS}\left(C_{3}^{(t)}\right)\right| \\
& =\left|\begin{array}{cc}
(\mu+8) I_{n-1}-8 J_{n-1} & -\left((n-1)^{2}+4\right) J_{(n-1) \times 1} \\
-\left((n-1)^{2}+4\right) J_{1 \times(n-1)} & \left.\left(\mu+2(n-1)^{2}\right) I_{1}-2(n-1)^{2} J_{1}\right)
\end{array}\right| .
\end{aligned}
$$

Now using Lemma 1, we get the desired result.

The helm $H_{n}$ is a graph obtained from a wheel $W_{n}$ with cetral vertex $c$, by attaching a pendant edge to each rim vertex of $W_{n}$. A closed helm is the graph with central vertex $c$, obtained from a helm by joining each pendant vertex to form a cycle.

Theorem 3. If the graph $H_{n}-c$ of order $2 n$ and size $2 n$ is a helm without central vertex, then

$$
P_{D S S\left(H_{n}-c\right)}(\mu)=(\mu+18)^{n-1}(\mu+2)^{n-1}\left\{\left[\mu-18(n-1)(\mu-2(n-1))-100 n^{2}\right\} .\right.
$$

Proof. The helm $H_{n}-c$ without central vertex is a graph of order $2 n$, which has two types of vertices. The $n$ vertices have degree 3 and the remaining $n$ vertices have degree 1 . Hence,

$$
\operatorname{DSS}\left(H_{n}-c\right)=\left[\begin{array}{cc}
18\left(J_{n}-I_{n}\right) & 10 J_{n \times n} \\
10 J_{n \times n} & 2\left(J_{n}-I_{n}\right)
\end{array}\right]
$$

Therefore,

$$
\begin{aligned}
P_{D S S\left(H_{n}-c\right)}(\mu) & =\left|\mu I-\operatorname{DSS}\left(H_{n}-c\right)\right| \\
& =\left|\begin{array}{cc}
(\mu+18) I_{n}-18 J_{n} & -10 J_{n \times n} \\
-10 J_{n \times n} & (\mu+2) I_{n}-2 J_{n}
\end{array}\right| .
\end{aligned}
$$

Now using Lemma 1, we get the desired result.

Theorem 4. If the graph $H_{n}^{\prime}-c$ of order $2 n$ and size $3 n$ is a closed helm without central vertex, then

$$
P_{D S S\left(H_{n}^{\prime}-c\right)}(\mu)=(\mu-54(2 n-1))(\mu+54)^{2 n-1} .
$$


InternationalJournalofAppliedEngineeringResearch,ISSN0973-4562Volume13,Number19(2018)pp.14060-14078

ResearchIndiaPublications, https://dx.doi.org/10.37622/IJAER/13.19.2018.14060-14078

Proof. The closed helm without central vertex $H_{n}^{\prime}-c$ is 3-regular graph with $2 n$ vertices. Hence, the result follows from Eq. (1).

Remark 5. The graph $W_{c}=C_{n} \circ K_{1}$ is called a Crown graph. The crown $W_{c}=C_{n} \circ K_{1}$ is a unicyclic graph of order $2 n$ and size $2 n$. That is the crown is obtained from the helm by removing the central vertex $c$.

The sunflower graph $S F_{n}$ is a graph obtained from a wheel with central vertex $c, n$-cycle $v_{0}, v_{1}, \ldots, v_{n-1}$ and additional $n$ vertices $w_{0}, w_{1}, \ldots, w_{n-1}$ where $w_{i}$ is joined by edges to $v_{i}, v_{i+1}$ for $i=0,1, \ldots, n-1$ where $i+1$ is taken modulo $n$.

Theorem 6. If the graph $S F_{n}-c$ of order $2 n$ and size $3 n$ is a sunflower graph without central vertex, then

$$
P_{D S S\left(S F_{n}-c\right)}(\mu)=(\mu+18)^{n-1}(\mu+8)^{n-1}\left\{[\mu-18(n-1)][\mu-8(n-1)]-169 n^{2}\right\} .
$$

Proof. The sunflower graph $S F_{n}-c$ without central vertex is a graph of order $2 n$, which has two types of vertices. The $n$ vertices have degree 3 and the remaining $n$ vertices have degree 2 . Hence,

$$
\operatorname{DSS}\left(S F_{n}-c\right)=\left[\begin{array}{cc}
18\left(J_{n}-I_{n}\right) & 13 J_{n \times n} \\
13 J_{n \times n} & 8\left(J_{n}-I_{n}\right)
\end{array}\right]
$$

Therefore,

$$
\begin{aligned}
P_{D S S\left(S F_{n}-c\right)}(\mu) & =\left|\mu I-\operatorname{DSS}\left(S F_{n}-c\right)\right| \\
& =\left|\begin{array}{cc}
(\mu+18) I_{n}-18 J_{n} & -13 J_{n \times n} \\
-13 J_{n \times n} & (\mu+8) I_{n}-8 J_{n}
\end{array}\right| .
\end{aligned}
$$

Now using Lemma 1, we get the desired result.

The lotus inside a circle $L C_{n}$ is a graph obtained from the cycle $C_{n}: w_{1} w_{2} \ldots w_{n} w_{1}$ and a star $K_{1, n}$ with central vertex $u$ and the end vertices $u_{i},(1 \leq n)$ by joining each $u_{i}$ to $w_{i}$ and $w_{i+1(\bmod n)}$.

Remark 7. The lotus inside a circle without central vertex is isomorphic to sunflower without the central vertex.

The double cone $D C_{n}=C_{n}+2 K_{1}$ is a graph with $n+2$ vertices and $3 n$ edges.

Theorem 8. If $D C_{n}$ is a double cone of order $n+2$ and size $3 n$, then

$$
P_{D S S\left(D C_{n}\right)}(\mu)=(\mu+32)^{n-1}\left(\mu+2 n^{2}\right)\left\{[\mu-32(n-1)]\left[\mu-2 n^{2}\right]-2 n\left(n^{2}+16\right)^{2}\right\} .
$$

Proof. The double cone is a graph of of order $n+2$ has two types of vertices. The $n$ vertices are of degree 4 and the remaining 2 vertices with degree $n$. Hence,

$$
\operatorname{DSS}\left(D C_{n}\right)=\left[\begin{array}{cc}
32\left(J_{n}-I_{n}\right) & \left(n^{2}+16\right) J_{n \times 2} \\
\left(n^{2}+16\right) J_{2 \times n} & 2 n^{2}\left(J_{2}-I_{2}\right)
\end{array}\right]
$$

Therefore,

$$
\begin{aligned}
P_{D S S\left(D C_{n}\right)}(\mu) & =\left|\mu I-D S S\left(D C_{n}\right)\right| \\
& =\left|\begin{array}{cc}
(\mu+32) I_{n}-32 J_{n} & -\left(n^{2}+16\right) J_{n \times 2} \\
-\left(n^{2}+16\right) J_{2 \times n} & \left(\mu+2 n^{2}\right) I_{2}-2 n^{2} J_{2}
\end{array}\right| .
\end{aligned}
$$

Now using Lemma 1, we get the expected result. 
InternationalJournalofAppliedEngineeringResearch,ISSN0973-4562Volume13,Number19(2018)pp.14060-14078 ResearchIndiaPublications, https://dx.doi.org/10.37622/IJAER/13.19.2018.14060-14078

The graph $S_{m} \times P_{2}$ (where $S_{m}$ is a star with $m+1$ vertices) is called a Book graph $B_{m} . B_{m}$ is a graph of order $2(m+1)$.

Theorem 9. If $B_{m}$ is a book graph, then

$$
P_{D S S\left(B_{m}\right)}(\mu)=(\mu+8)^{2 m-1}\left(\mu+2(m+1)^{2}\right)\left\{[\mu-8(2 m-1)]\left[\mu-2(m+1)^{2}\right]-4 m\left((m+1)^{2}+4\right)^{2}\right\} .
$$

Proof. The Book graph $B_{m}$ has two types of vertices. The $2 m$ vertices with degree 2 and 2 vertices are with degree $m+1$. Hence,

$$
\operatorname{DSS}\left(B_{m}\right)=\left[\begin{array}{cc}
8\left(J_{2 m}-I_{2 m}\right) & \left(2^{2}+(m+1)^{2}\right) J_{2 m \times 2} \\
\left(2^{2}+(m+1)^{2}\right) J_{2 \times 2 m} & 2(m+1)^{2}\left(J_{2}-I_{2}\right)
\end{array}\right]
$$

Therefore,

$$
\begin{aligned}
P_{D S S\left(B_{m}\right)}(\mu) & =\left|\mu I-D S S\left(B_{m}\right)\right| \\
& =\left|\begin{array}{cc}
(\mu+8) I_{2 m}-8 J_{2 m} & -\left((m+1)^{2}+4\right) J_{2 m \times 2} \\
-\left((m+1)^{2}+4\right) J_{2 \times 2 m} & \left(\mu+2(m+1)^{2}\right) I_{2}-2(m+1)^{2} J_{2}
\end{array}\right| .
\end{aligned}
$$

Now using Lemma 1, we get the desired result.

The graph $P_{n} \times P_{2}$ is called a Ladder graph $L_{n}$.

Theorem 10. If $L_{n}$ is a ladder graph, then

$$
P_{D S S\left(L_{n}\right)}(\mu)=(\mu+18)^{2 n-5}(\mu+8)^{3}\{[\mu-18(2 n-5)](\mu-24)-676(2 n-4)\} .
$$

Proof. The Ladder graph $L n$ is a graph of order $2 n$ and has two types of vertices. The 4 vertices have degree 2 and $2 n-4$ vertices have degree 3 . Hence,

$$
\operatorname{DSS}\left(L_{n}\right)=\left[\begin{array}{cc}
18\left(J_{2 n-4}-I_{2 n-4}\right) & 13 J_{(2 n-4) \times 4} \\
13 J_{4 \times(2 n-4)} & 8\left(J_{4}-I_{4}\right)
\end{array}\right]
$$

Therefore,

$$
\begin{aligned}
P_{D S S\left(L_{n}\right)}(\mu) & =\left|\mu I-\operatorname{DSS}\left(L_{n}\right)\right| \\
& =\left|\begin{array}{cc}
(\mu+18) I_{2 n-4}-18 J_{2 n-4} & -13 J_{(2 n-4) \times 4} \\
-13 J_{4 \times(2 n-4)} & (\mu+8) I_{4}-8 J_{4}
\end{array}\right| .
\end{aligned}
$$

Now using Lemma 1, we get the expected result.

The prism $\Pi_{n}=C_{n} \times P_{2}$ is a 3-regular graph of order $2 n$ and size $3 n$

Theorem 11. If the prism $\Pi_{n}$ is a graph of order $2 n$ and size $3 n$, then

$$
P_{D S S\left(\Pi_{n}\right)}(\mu)=(\mu-18(2 n-1))(\mu+18)^{2 n-1} .
$$

Proof. The prism $\Pi_{n}$ is 3-regular graph with $2 n$ vertices. Hence, the result follows from Eq. (1).

The point splitting graph $S^{\prime}(G)$ of a graph $G$ is obtained from a graph $G$ by adding for each vertex $v$ of $G$ a new vertex $v^{\prime}$ so that $v^{\prime}$ is adjacent to every vertex that is adjacent to $v$. 
InternationalJournalofAppliedEngineeringResearch,ISSN0973-4562Volume13,Number19(2018)pp.14060-14078 ResearchIndiaPublications, https://dx.doi.org/10.37622/IJAER/13.19.2018.14060-14078

Theorem 12. If $G$ is an r-regular graph of order $n$, then

$$
\left.P_{D S S\left(S^{\prime}(G)\right)}(\mu)=\left(\mu+8 r^{2}\right)^{n-1}\left(\mu+2 r^{2}\right) n-1[\mu-8(n-1)]\left[\mu-2(n-1) r^{2}\right]-25 r^{4} n^{2}\right\} .
$$

Proof. The point splitting graph of an $r$-regular graph is a graph of order $2 n$ and has two types of vertices. The $n$ vertices have degree $2 r$ and the remaining $n$ vertices have degree $r$. Hence,

$$
\operatorname{DSS}\left(S^{\prime}(G)\right)=\left[\begin{array}{cc}
2(2 r)^{2}\left(J_{n}-I_{n}\right) & \left((2 r)^{2}+r^{2}\right) J_{n \times n} \\
\left((2 r)^{2}+r^{2}\right) J_{n \times n} & 2 r^{2}\left(J_{n}-I_{n}\right)
\end{array}\right]
$$

Therefore,

$$
\begin{aligned}
P_{D S S\left(S^{\prime}(G)\right)}(\mu) & =\left|\mu I-\operatorname{DSS}\left(S^{\prime}(G)\right)\right| \\
& =\left|\begin{array}{cc}
\left(\mu+8 r^{2}\right) I_{n}-8 r^{2} J_{n} & -5 r^{2} J_{n \times m} \\
-5 r^{2} J_{m \times n} & 2 r^{2} I_{m}-2 r^{2} J_{m}
\end{array}\right| .
\end{aligned}
$$

Now by using Lemma 1, we get the required result.

The line splitting graph[14] $L_{s}(G)$ of a graph $G$ is a graph with vertex set $E(G) \cup E_{1}(G)$ with two vertices adjacent if they correspond to adjacent edges of $G$ or one corresponds to an element $e_{i}^{\prime}$ of $E_{1}(G)$ and the other to an element $e_{j}$ of $E(G)$ where $e_{j}$ is in $N\left(e_{i}\right)$.

Theorem 13. If $G$ is an $r$-regular graph of order $n$ and size $m$, then

$$
\begin{aligned}
P_{D S S\left(L_{s}(G)\right)}(\mu)= & {\left[\mu+2(2 r-2)^{2}\right]^{m-1}\left[\mu+2(4 r-4)^{2}\right]^{m-1}\left\{\left[\mu-2(m-1)(2 r-2)^{2}\right][\mu\right.} \\
& \left.\left.-2(m-1)(4 r-4)^{2}\right]-m^{2}\left[(2 r-2)^{2}+(4 r-4)^{2}\right]^{2}\right\} .
\end{aligned}
$$

Proof. The line splitting graph of an $r$-regular graph of size $m$ is a graph of order $2 m . L_{s}(G)$ has 2 types of vertices. The $m$ vertices have degree $2 r-2$ and the remaining $m$ vertices have degree $4 r-4$. Hence,

$$
\operatorname{DSS}\left(L_{s}(G)\right)=\left[\begin{array}{cc}
2(2 r-2)^{2}\left(J_{m}-I_{m}\right) & {\left[(2 r-2)^{2}+(4 r-4)^{2}\right] J_{m \times m}} \\
{\left[(2 r-2)^{2}+(4 r-4)^{2}\right] J_{m \times m}} & 2(4 r-4)^{2}\left(J_{m}-I_{m}\right)
\end{array}\right]
$$

Therefore,

$$
\begin{gathered}
P_{D S S\left(L_{s}(G)\right)}(\mu)=\left|\mu I-\operatorname{DSS}\left(L_{s}(G)\right)\right| \\
=\left|\begin{array}{cc}
{\left[\mu+2(2 r-2)^{2}\right] I_{m}-2(2 r-2)^{2} J_{m}} & -\left[(2 r-2)^{2}+(4 r-4)^{2}\right] J_{m \times m} \\
-\left[(2 r-2)^{2}+(4 r-4)^{2}\right] J_{m \times m} & \left(\mu+2(4 r-4)^{2}\right) I_{m}-2(4 r-4)^{2} J_{m}
\end{array}\right| .
\end{gathered}
$$

Next by using Lemma 1, we get the desired result.

Let $G=(V, E)$ be a graph with $V=S_{1} \cup S_{2} \cup \cdots \cup S_{t} \cup T$ where each $S_{i}$ is a set of vertices having at least two vertices and having the same degree and $T=V-\cup_{i=1}^{t} S_{i}$. The degree splitting graph [17] $D S(G)$ is obtained from $G$ by adding vertices $w_{1}, w_{2}, \ldots, w_{t}$ and joining $w_{i}$ to each vertex of $S_{i}(1 \leq i \leq t)$.

Theorem 14. If $G$ is an $r$-regular graph of order $n$, then

$$
P_{D S S(D S(G))}(\mu)=\left(\mu+2(r+1)^{2}\right)^{n-1}\left\{\left(\mu-2(n-1)(r+1)^{2}\right)-n\left[(r+1)^{2}+n^{2}\right]^{2}\right\} .
$$


InternationalJournalofAppliedEngineeringResearch,ISSN0973-4562Volume13,Number19(2018)pp.14060-14078

ResearchIndiaPublications, https://dx.doi.org/10.37622/IJAER/13.19.2018.14060-14078

Proof. The degree splitting graph $D S(G)$ of a $r$-regular graph $G$ with $n$ vertices is graph, which has two types of vertices. The $n$ vertices with degree $(r+1)$ and the remaining 1 vertex is of degree $n$. Hence,

$$
\operatorname{DSS}(D S(G))=\left[\begin{array}{cc}
2(r+1)^{2}\left(J_{n}-I_{n}\right) & \left((r+1)^{2}+n^{2}\right) J_{n \times 1} \\
\left((r+1)^{2}+n^{2}\right) J_{1 \times n} & 2 n^{2}\left(J_{1}-I_{1}\right)
\end{array}\right]
$$

Therefore,

$$
\begin{aligned}
P_{D S S(D S(G))}(\mu) & =|\mu I-\operatorname{DSS}(D S(G))| \\
& =\left|\begin{array}{cc}
\left(\mu+2(r+1)^{2}\right) I_{n}-2(r+1)^{2} J_{n} & -\left((r+1)^{2}+n^{2}\right) J_{n \times 1} \\
-\left((r+1)^{2}+n^{2}\right) J_{1 \times n} & 0
\end{array}\right| .
\end{aligned}
$$

By using Lemma 1, we get the desired result.

The book with triangular pages $B_{t}=P_{2}+t K_{1}$ is a graph with $(n+2)$ vertices and $2 n+1$ edges.

Theorem 15. If $B_{t}$ is a book with triangular pages of order $(t+2)$ and size $2 t+1$, then

$$
P_{D S S\left(B_{t}\right)}(\mu)=(\mu+8)^{t-1}\left(\mu+2(t+1)^{2}\right)\left\{(\mu-8(t-1))\left[\mu-2(t+1)^{2}\right]-2 t\left((t+1)^{2}+4\right)^{2}\right\} .
$$

Proof. The book $B_{t}$ with triangular pages has two types of vertices. The $t$ vertices are of degree 2 and the remaining 2 vertices are of degree $t+1$. Hence,

$$
\operatorname{DSS}\left(B_{t}\right)=\left[\begin{array}{cc}
8\left(J_{t}-I_{t}\right) & \left(2^{2}+(t+1)^{2}\right) J_{t \times 2} \\
\left(2^{2}+(t+1)^{2}\right) J_{2 \times t} & 2(t+1)^{2}\left(J_{2}-I_{2}\right)
\end{array}\right]
$$

Therefore,

$$
\begin{aligned}
P_{D S S\left(B_{t}\right)}(\mu) & =\left|\mu I-\operatorname{DSS}\left(B_{t}\right)\right| \\
& =\left|\begin{array}{cc}
(\mu+8) I_{t}-8 J_{t} & -\left((t+1)^{2}+4\right) J_{t \times 2} \\
-\left((t+1)^{2}+4\right) J_{2 \times t} & \left(\mu+2(t+1)^{2}\right) I_{2}-2(t+1)^{2} J_{2}
\end{array}\right| .
\end{aligned}
$$

Now by using Lemma 1, we get the required result.

The shadow graph $D_{2}(G)$ of a connected graph $G$ is constructed by taking two copies of $G$, say $G^{\prime}, G^{\prime \prime}$ and joining each vertex $v^{\prime}$ in $G^{\prime}$ to the neighbors of the corresponding vertex $v^{\prime \prime}$ in $G^{\prime \prime}$.

Theorem 16. If $G$ is an $r$-regular graph of order $n$, then

$$
P_{D S S\left(D_{2}(G)\right)}(\mu)=\left[\mu-8 r^{2}(2 n-1)\right]\left(\mu+8 r^{2}\right)^{2 n-1} .
$$

Proof. The shadow graph $D_{2}(G)$ of an $r$-regular graph $G$ is a $2 r$-regular graph with $2 n$ vertices. Hence the result follows from Eq. (1).

Theorem 17. If $H$ is a graph having $n$ vertices of degree $x$ and $m$ vertices of degree $y$, then

$$
P_{D S S\left(D_{2}(H)\right)}(\mu)=\left(\mu+8 x^{2}\right)^{2 n-1}\left(\mu+8 y^{2}\right)^{2 m-1}\left\{\left[\mu-8 x^{2}(2 n-1)\right]\left[\mu-8 y^{2}(2 m-1)-4 m n\left(4 x^{2}+4 y^{2}\right)^{2}\right\} .\right.
$$

Proof. The shadow graph $D_{2}(H)$ is a graph, which has two types of vertices. The $2 n$ vertices with degree $2 x$ and the remaining $2 m$ vertices are of degree $2 y$ since $H$ has $n$ vertices with degree $x$ and $m$ vertices with degree $y$. Hence,

$$
\operatorname{DSS}\left(D_{2}(H)\right)=\left[\begin{array}{cc}
2(2 x)^{2}\left(J_{2 n}-I_{2 n}\right) & \left((2 x)^{2}+(2 y)^{2}\right) J_{2 n \times 2 m} \\
\left((2 x)^{2}+(2 y)^{2}\right) J_{2 m \times 2 n} & 2(2 y)^{2}\left(J_{2 m}-I_{2 m}\right)
\end{array}\right]
$$


InternationalJournalofAppliedEngineeringResearch,ISSN0973-4562Volume13,Number19(2018)pp.14060-14078 ResearchIndiaPublications, https://dx.doi.org/10.37622/IJAER/13.19.2018.14060-14078

Therefore,

$$
\begin{aligned}
P_{D S S\left(D_{2}(H)\right)}(\mu) & =\left|\mu I-\operatorname{DSS}\left(D_{2}(H)\right)\right| \\
& =\left|\begin{array}{cc}
\left(\mu+2(2 x)^{2}\right) I_{2 n}-2(2 x)^{2} J_{2 n} & -\left((2 x)^{2}+(2 y)^{2}\right) J_{2 n \times 2 m} \\
-\left((2 x)^{2}+(2 y)^{2}\right) J_{2 m \times 2 n} & \left(\mu+2(2 y)^{2}\right) I_{2 m}-2(2 y)^{2} J_{2 m}
\end{array}\right| .
\end{aligned}
$$

By using Lemma 1, we get the desired result.

The triangular snake $T_{n}$ is a graph obtained from the path $P_{n}$ by replacing each edge of the path by a triangle $C_{3}$.

Theorem 18. If $T_{n}$ is a triangular snake of order $2 n-1$, then

$$
P_{D S S\left(T_{n}\right)}(\mu)=(\mu+8)^{n}(\mu+32)^{n-3}\{(\mu-8 n)(\mu-32(n-3))-400(n+1)(n-2)\} .
$$

Proof. The graph triangular snake $T_{n}$ has two types of vertices. The $n+1$ vertices with degree 2 and the remaining $n-2$ vertices are of degree 4 . Hence,

$$
\operatorname{DSS}\left(T_{n}\right)=\left[\begin{array}{cc}
8\left(J_{n+1}-I_{n+1}\right) & 20 J_{(n+1) \times(n-2)} \\
20 J_{(n-2) \times(n+1)} & 32\left(J_{n-2}-I_{n-2}\right)
\end{array}\right]
$$

Therefore,

$$
\begin{aligned}
P_{D S S\left(T_{n}\right)}(\mu) & =\left|\mu I-\operatorname{DSS}\left(T_{n}\right)\right| \\
& =\left|\begin{array}{cc}
(\mu+8) I_{n+1}-8 J_{n+1} & -20 J_{(n+1) \times(n-2)} \\
-20 J_{(n-2) \times(n+1)} & (\mu+32) I_{n-2}-32 J_{n-2}
\end{array}\right| .
\end{aligned}
$$

By using Lemma 1, we get the desired result.

The alternate triangular snake $A\left(T_{n}\right)$ is obtained from a path $v_{1} v_{2} \ldots v_{n}$ by joining $v_{i}$ and $v_{i+1}$ (alternatively) to new vertex $v_{i}$. That is every alternate edge of a path is replaced by $C_{3}$.

Theorem 19. If $A\left(T_{2 n}\right),(n \geq 2)$ is an alternate triangular snake of order $3 n$, then

$$
P_{D S S\left(A\left(T_{2 n}\right)\right)}(\mu)=(\mu+8)^{n+1}(\mu+18)^{2 n-3}\{(\mu-8(n+1))(\mu-18(2 n-3)) 169(n+2)(2 n-2)\} .
$$

Proof. The alternate triangular snake $A\left(T_{2 n}\right)$ is a graph, which has two types of vertices. The $n+2$ vertices have degree 2 and the remaining $2 n-2$ vertices have degree 3 . Hence,

$$
\operatorname{DSS}\left(A\left(T_{2 n}\right)\right)=\left[\begin{array}{cc}
8\left(J_{n+2}-I_{n+2}\right) & 13 J_{(n+2) \times(2 n-2)} \\
13 J_{(2 n-2) \times(n+2)} & 18\left(J_{2 n-2}-I_{2 n-2}\right)
\end{array}\right]
$$

Therefore,

$$
\begin{aligned}
P_{D S S\left(A\left(T_{2 n}\right)\right)}(\mu) & =\left|\mu I-\operatorname{DSS}\left(T_{2 n}\right)\right| \\
& =\left|\begin{array}{cc}
(\mu+8) I_{n+2}-8 J_{n+2} & -13 J_{(n+2) \times(2 n-2)} \\
-13 J_{(2 n-2) \times(n+2)} & (\mu+18) I_{2 n-2}-18 J_{2 n-2}
\end{array}\right| .
\end{aligned}
$$

By using Lemma 1, we get the desired result.

The quadrilateral snake $Q_{n}$ is obtained from the path $P_{n}$ by replacing each edge of the path by a quadrilateral $C_{4}$. 
InternationalJournalofAppliedEngineeringResearch,ISSN0973-4562Volume13,Number19(2018)pp.14060-14078 ResearchIndiaPublications, https://dx.doi.org/10.37622/IJAER/13.19.2018.14060-14078

Theorem 20. If $Q_{n}$ is a quadrilateral snake of order $3 n-2$, then

$$
P_{D S S\left(Q_{n}\right)}(\mu)=(\mu+8)^{2 n-1}(\mu+32)^{n-3}\{(\mu-8(2 n-1))(\mu-32(n-3))-800 n(n-2)\} .
$$

Proof. The quadrilateral snake $Q_{n}$ is a graph, which has two types of vertices. The $2 n$ vertices have degree 2 and the remaining $n-2$ vertices have degree 4 . Hence,

$$
\operatorname{DSS}\left(Q_{n}\right)=\left[\begin{array}{cc}
8\left(J_{2 n}-I_{2 n}\right) & 20 J_{2 n \times(n-2)} \\
20 J_{(n-2) \times 2 n} & 32\left(J_{n-2}-I_{n-2}\right)
\end{array}\right]
$$

Therefore,

$$
\begin{aligned}
P_{D S S\left(Q_{n}\right)}(\mu) & =\left|\mu I-\operatorname{DSS}\left(Q_{n}\right)\right| \\
& =\left|\begin{array}{cc}
(\mu+8) I_{2 n}-8 J_{2 n} & -20 J_{2 n \times(n-2)} \\
-20 J_{(n-2) \times 2 n} & (\mu+32) I_{n-2}-32 J_{n-2}
\end{array}\right| .
\end{aligned}
$$

By using Lemma 1, we get the desired result.

The alternate quadrilateral snake $A\left(Q_{n}\right)$ is obtained from a path $v_{1} v_{2} \ldots v_{n}$ by joining $v_{i} v_{i+1}$ (alternatively) to new vertices $v_{i}, w_{i}$ respectively and then joining $v_{i}$ and $w_{i}$. That is every alternate edge of a path is replaced by a cycle $C_{4}$.

Theorem 21. If $A\left(Q_{2 n}\right),(n \geq 2)$ is a quadrilateral snake of order $4 n$, then

$$
P_{D S S\left(A\left(Q_{2 n}\right)\right)}(\mu)=(\mu+8)^{2 n+1}(\mu+18)^{2 n-3}\{(\mu-8(2 n+1))(\mu-18(2 n-3))-169(2 n+1)(2 n-3)\} .
$$

Proof. The alternate quadrilateral snake $A\left(Q_{2 n}\right)$ is a graph, which has two types of vertices. The $2 n+2$ vertices with degree 2 and the remaining $2 n-2$ vertices are of degree 3 . Hence,

$$
\operatorname{DSS}\left(A\left(Q_{2 n}\right)\right)=\left[\begin{array}{cc}
8\left(J_{2 n+2}-I_{2 n+2}\right) & 13 J_{(2 n+2) \times(2 n-2)} \\
13 J_{(2 n-2) \times(2 n+2)} & 18\left(J_{(2 n-2)}-I_{(2 n-2)}\right)
\end{array}\right]
$$

Therefore,

$$
\begin{aligned}
P_{D S S\left(A\left(Q_{2 n}\right)\right)}(\mu) & =\left|\mu I-\operatorname{DSS}\left(A\left(Q_{2 n}\right)\right)\right| \\
& =\left|\begin{array}{cc}
(\mu+8) I_{2 n+2}-8 J_{2 n+2} & -13 J_{(2 n+2) \times(2 n-2)} \\
-13 J_{(2 n-2) \times(2 n+2)} & (\mu+18) I_{2 n-2}-18 J_{2 n-2}
\end{array}\right| .
\end{aligned}
$$

By using Lemma 1, we get the desired result.

\section{DEGREE SQUARE SUM POLYNOMIALS OF GENERALIZED $x y z$-POINT-LINE TRANSFORMATION GRAPHS $T^{x y z}(G)$}

The procedure of obtaining a new graph from a given graph by using incidence (or nonincidence) relation between vertex and an edge and an adjacency (or nonadjacency) relation between two vertices or two edges of a graph is known as Graph Transformation and the graph obtained by doing so is called a Transformation graph. For a graph $G=(V, E)$, let $G^{0}$ be the graph with $V\left(G^{0}\right)=V(G)$ and with no edges, $G^{1}$ the complete graph with $V\left(G^{1}\right)=V(G), G^{+}=G$, and $G^{-}=\bar{G}$. Let $\mathcal{G}$ denotes the set of simple graphs. The following graph operations depending on $x, y, z \in\{0,1,+,-\}$ induce functions $T^{x y z}: \mathcal{G} \rightarrow \mathcal{G}$. These operations were introduced by Deng et al. in [9]. They called these resulting 
InternationalJournalofAppliedEngineeringResearch,ISSN0973-4562Volume13,Number19(2018)pp.14060-14078

ResearchIndiaPublications, https://dx.doi.org/10.37622/IJAER/13.19.2018.14060-14078

graphs as $x y z$-transformations of $G$, denoted by $T^{x y z}(G)=G^{x y z}$ and studied the Laplacian characteristic polynomials and some other Laplacian parameters of $x y z$-transformations of an $r$-regular graph $G$. In [2], Wu Bayoindureng et al. introduced the total transformation graphs and studied the basic properties of total transformation graphs. Motivated by this, Basavanagoud [3] studied the basic properties of the $x y z$-transformation graphs and called them $x y z$-point-line transformation graphs by changing the notation of $x y z$-transformations of a graph $G$ as $T^{x y z}(G)$ to avoid confusion between parent graph $G$ and its $x y z$-transformations.

Definition 22. [9] Given a graph $G(n, m)$ with vertex set $V(G)$ and edge set $E(G)$ and three variables $x, y, z \in\{0,1,+,-\}$, the xyz-point-line transformation graph $T^{x y z}(G)$ of $G$ is the graph with vertex set $V\left(T^{x y z}(G)\right)=V(G) \cup E(G)$ i.e $|V(G)|=n+m$ and the edge set $E\left(T^{x y z}(G)\right)=E\left((G)^{x}\right) \cup E\left((L(G))^{y}\right) \cup E(W)$ where $W=S(G)$ if $z=+, W=\bar{S}(G)$ if $z=-, W$ is the graph with $V(W)=V(G) \cup E(G)$ and with no edges if $z=0$ and $W$ is the complete bipartite graph with parts $V(G)$ and $E(G)$ if $z=1$.

Since there are 64 distinct 3-permutations of $\{0,1,+,-\}$, thus obtained 64 kinds of generalized $x y z$-point-line transformation graphs. There are 16 different graphs for each case when $z=0, z=1, z=+, z=-$.

The following Propositions are useful for proving forthcoming theorems.

Proposition 23. [5] If $G$ is a graph of order $n$ and size $m$ and let $v$ be a point-vertex of $T^{x y z}(G)$ corresponding to a vertex $v$ of $G$, then

(i)

$$
d_{T^{x y+}(G)}(v)= \begin{cases}d_{G}(v) & \text { if } x=0, y \in\{0,1,+,-\} \\ n-1+d_{G}(v) & \text { if } x=1, y \in\{0,1,+,-\} \\ 2 d_{G}(v) & \text { if } x=+, y \in\{0,1,+,-\} \\ n-1 & \text { if } x=-, y \in\{0,1,+,-\}\end{cases}
$$

(ii)

$$
d_{T^{x y}-(G)}(v)= \begin{cases}m-d_{G}(v) & \text { if } x=0, y \in\{0,1,+,-\} \\ n+m-1-d_{G}(v) & \text { if } x=1, y \in\{0,1,+,-\} \\ m & \text { if } x=+, y \in\{0,1,+,-\} \\ n+m-1-2 d_{G}(v) & \text { if } x=-, y \in\{0,1,+,-\}\end{cases}
$$

Proposition 24. [5] If $G$ is a graph of order $n$ and size $m$ and let $e$ be the line-vertex of $T^{x y z}(G)$ corresponding to an edge $e$ of $G$, then

(i)

$$
d_{T^{x y+}(G)}(e)= \begin{cases}2 & \text { if } y=0, x \in\{0,1,+,-\} \\ m+1 & \text { if } y=1, x \in\{0,1,+,-\} \\ 2+d_{G}(e) & \text { if } y=+, x \in\{0,1,+,-\} \\ m+1-d_{G}(e) & \text { if } y=-, x \in\{0,1,+,-\}\end{cases}
$$

(ii)

$$
d_{T^{x y-}(G)}(e)= \begin{cases}n-2 & \text { if } y=0, x \in\{0,1,+,-\} \\ n+m-3 & \text { if } y=1, x \in\{0,1,+,-\} \\ n-2+d_{G}(e) & \text { if } y=+, x \in\{0,1,+,-\} \\ n+m-3-d_{G}(e) & \text { if } y=-, x \in\{0,1,+,-\}\end{cases}
$$

Theorem 25. If $G$ is an $r$-regular graph of order $n$ and size $m$, then

$$
\begin{aligned}
P_{D S S\left(T^{10+}(G)\right)}(\mu)= & {\left[\mu+2(n-1+r)^{2}\right]^{n-1}[\mu+8]^{m-1}\left(\left[\mu-2(n-1)(n-1+r)^{2}\right][\mu-8(m-1)]\right.} \\
& \left.-m n\left((n-1+r)^{2}+2^{2}\right)^{2}\right) .
\end{aligned}
$$


InternationalJournalofAppliedEngineeringResearch,ISSN0973-4562Volume13,Number19(2018)pp.14060-14078 ResearchIndiaPublications, https://dx.doi.org/10.37622/IJAER/13.19.2018.14060-14078

Proof. The graph $T^{10+}(G)$ of an $r$-regular graph $G$ has two types of vertices. From Propositions 23 and 24, the $n$ vertices have degree $n-1+r$ and $m$ vertices have degree 2 . Hence,

$$
D S S\left(T^{10+}(G)\right)=\left[\begin{array}{cc}
2(n-1+r)^{2}\left(J_{n}-I_{n}\right) & \left((n-1+r)^{2}+4\right) J_{n \times m} \\
\left((n-1+r)^{2}+4\right) J_{m \times n} & 8\left(J_{m}-I_{m}\right)
\end{array}\right]
$$

Therefore,

$$
\begin{aligned}
P_{D S S\left(T^{10+}(G)\right)}(\mu) & =\left|\mu I-D S S\left(T^{10+}(G)\right)\right| \\
& =\left|\begin{array}{cc}
\left(\mu+2(n-1+r)^{2}\right) I_{n}-2(n-1+r)^{2} J_{n} & -\left((n-1+r)^{2}+4\right) J_{n \times m} \\
-\left((n-1+r)^{2}+4\right) J_{m \times n} & (\mu+8) I_{m}-8 J_{m}
\end{array}\right| .
\end{aligned}
$$

Now using Lemma 1, we get the desired result.

Theorem 26. If $G$ is an $r$-regular graph of order $n$ and size $m$, then

$$
\begin{aligned}
P_{D S S\left(T^{-0+}(G)\right)}(\mu)= & {\left[\mu+2(n-1)^{2}\right]^{n-1}[\mu+8]^{m-1}\left(\left[\mu-2(n-1)^{3}\right][\mu-8(m-1)]\right.} \\
& \left.-m n\left((n-1)^{2}+2^{2}\right)^{2}\right) .
\end{aligned}
$$

Proof. The graph $T^{-0+}(G)$ of an $r$-regular graph $G$ has two types of vertices. From Propositions 23 and 24, the $n$ vertices have degree $n-1$ and $m$ vertices have degree 2 . Hence,

$$
\operatorname{DSS}\left(T^{-0+}(G)\right)=\left[\begin{array}{cc}
2(n-1)^{2}\left(J_{n}-I_{n}\right) & \left((n-1)^{2}+4\right) J_{n \times m} \\
\left((n-1)^{2}+4\right) J_{m \times n} & 8\left(J_{m}-I_{m}\right)
\end{array}\right]
$$

Therefore,

$$
\begin{aligned}
P_{D S S\left(T^{-0+}(G)\right)}(\mu) & =\left|\mu I-D S S\left(T^{-0+}(G)\right)\right| \\
& =\left|\begin{array}{cc}
\left(\mu+2(n-1)^{2}\right) I_{n}-2(n-1)^{2} J_{n} & -\left((n-1)^{2}+4\right) J_{n \times m} \\
-\left((n-1)^{2}+4\right) J_{m \times n} & (\mu+8) I_{m}-8 J_{m}
\end{array}\right| .
\end{aligned}
$$

Now using Lemma 1, we get the desired result.

Theorem 27. If $G$ is an $r$-regular graph of order $n$ and size $m$, then

$$
\begin{aligned}
P_{D S S\left(T^{01+}(G)\right)}(\mu)= & {\left[\mu+2 r^{2}\right]^{n-1}\left[\mu+2(m+1)^{2}\right]^{m-1}\left(\left[\mu-2(n-1) r^{2}\right]\left[\mu-2(m-1)(m+1)^{2}\right]\right.} \\
& \left.-m n\left(r^{2}+(m+1)^{2}\right)^{2}\right) .
\end{aligned}
$$

Proof. The graph $T^{01+}(G)$ of an $r$-regular graph $G$ has two types of vertices. From Propositions 23 and 24, the $n$ vertices have degree $r$ and $m$ vertices have degree $m+1$. Hence,

$$
\operatorname{DSS}\left(T^{01+}(G)\right)=\left[\begin{array}{cc}
2 r^{2}\left(J_{n}-I_{n}\right) & \left(r^{2}+(m+1)^{2}\right) J_{n \times m} \\
\left(r^{2}+(m+1)^{2}\right) J_{m \times n} & 2(m+1)^{2}\left(J_{m}-I_{m}\right)
\end{array}\right]
$$

Therefore,

$$
\begin{aligned}
P_{D S S\left(T^{01+}(G)\right)}(\mu) & =\left|\mu I-\operatorname{DSS}\left(T^{01+}(G)\right)\right| \\
& =\left|\begin{array}{cc}
\left(\mu+2 r^{2}\right) I_{n}-2 r^{2} J_{n} & -\left(r^{2}+(m+1)^{2}\right) J_{n \times m} \\
-\left(r^{2}+(m+1)^{2}\right) J_{m \times n} & \left(\mu+2(m+1)^{2}\right) I_{m}-2(m+1)^{2} J_{m}
\end{array}\right| .
\end{aligned}
$$

Now using Lemma 1, we get the desired result. 
InternationalJournalofAppliedEngineeringResearch,ISSN0973-4562Volume13,Number19(2018)pp.14060-14078 ResearchIndiaPublications, https://dx.doi.org/10.37622/IJAER/13.19.2018.14060-14078

Theorem 28. If $G$ is an $r$-regular graph of order $n$ and size $m$, then

$$
\begin{aligned}
P_{D S S\left(T^{11+}(G)\right)}(\mu)= & {\left[\mu+2(n-1+r)^{2}\right]^{n-1}\left[\mu+2(m+1)^{2}\right]^{m-1}\left(\left[\mu-2(n-1)(n-1+r)^{2}\right][\mu\right.} \\
& \left.\left.-2(m-1)(m+1)^{2}\right]-m n\left((n-1+r)^{2}+(m+1)^{2}\right)^{2}\right) .
\end{aligned}
$$

Proof. The graph $T^{11+}(G)$ of an $r$-regular graph $G$ has two types of vertices. From Propositions 23 and 24, the $n$ vertices have degree $n-1+r$ and $m$ vertices have degree $m+1$. Hence,

$$
\operatorname{DSS}\left(T^{11+}(G)\right)=\left[\begin{array}{cc}
2(n-1+r)^{2}\left(J_{n}-I_{n}\right) & \left((n-1+r)^{2}+(m+1)^{2}\right) J_{n \times m} \\
\left((n-1+r)^{2}+(m+1)^{2}\right) J_{m \times n} & 2(m+1)^{2}\left(J_{m}-I_{m}\right)
\end{array}\right]
$$

Therefore,

$$
\begin{aligned}
P_{D S S\left(T^{11+}(G)\right)}(\mu) & =\left|\mu I-D S S\left(T^{11+}(G)\right)\right| \\
& =\left|\begin{array}{cc}
\left(\mu+2(n-1+r)^{2}\right) I_{n}-2(n-1+r)^{2} J_{n} & -\left((n-1+r)^{2}+(m+1)^{2}\right) J_{n \times m} \\
-\left((n-1+r)^{2}+(m+1)^{2}\right) J_{m \times n} & \left(\mu+2(m+1)^{2}\right) I_{m}-2(m+1)^{2} J_{m}
\end{array}\right| .
\end{aligned}
$$

Now using Lemma 1, we get the desired result.

Theorem 29. If $G$ is an $r$-regular graph of order $n$ and size $m$, then

$$
\begin{aligned}
P_{D S S\left(T^{+1+}(G)\right)}(\mu)= & {\left[\mu+8 r^{2}\right]^{n-1}\left[\mu+2(m+1)^{2}\right]^{m-1}\left(\left[\mu-8(n-1) r^{2}\right]\left[\mu-2(m-1)(m+1)^{2}\right]\right.} \\
& \left.-m n\left(4 r^{2}+(m+1)^{2}\right)^{2}\right) .
\end{aligned}
$$

Proof. The graph $T^{+1+}(G)$ of an $r$-regular graph $G$ has two types of vertices. From Propositions 23 and 24, the $n$ vertices have degree $2 r$ and $m$ vertices have degree $m+1$. Hence,

$$
\operatorname{DSS}\left(T^{+1+}(G)\right)=\left[\begin{array}{cc}
8 r^{2}\left(J_{n}-I_{n}\right) & \left(4 r^{2}+(m+1)^{2}\right) J_{n \times m} \\
\left(4 r^{2}+(m+1)^{2}\right) J_{m \times n} & 2(m+1)^{2}\left(J_{m}-I_{m}\right)
\end{array}\right]
$$

Therefore,

$$
\begin{aligned}
P_{D S S\left(T^{+1+}(G)\right)}(\mu) & =\left|\mu I-\operatorname{DSS}\left(T^{+1+}(G)\right)\right| \\
& =\left|\begin{array}{cc}
\left(\mu+8 r^{2}\right) I_{n}-8 r^{2} J_{n} & -\left(4 r^{2}+(m+1)^{2}\right) J_{n \times m} \\
-\left(4 r^{2}+(m+1)^{2}\right) J_{m \times n} & \left(\mu+2(m+1)^{2}\right) I_{m}-2(m+1)^{2} J_{m}
\end{array}\right| .
\end{aligned}
$$

Now using Lemma 1, we get the desired result.

Theorem 30. If $G$ is an $r$-regular graph of order $n$ and size $m$, then

$$
\begin{aligned}
P_{D S S\left(T^{1++}(G)\right)}(\mu)= & {\left[\mu+2(n-1+r)^{2}\right]^{n-1}\left[\mu+2 r^{2}\right]^{m-1}\left(\left[\mu-2(n-1)(n-1+r)^{2}\right]\left[\mu-2(m-1) r^{2}\right]\right.} \\
& \left.-m n\left((n-1+r)^{2}+r^{2}\right)^{2}\right) .
\end{aligned}
$$

Proof. The graph $T^{1++}(G)$ of an $r$-regular graph $G$ has two types of vertices. From Propositions 23 and 24, the $n$ vertices have degree $n-1+r$ and $m$ vertices have degree $r$. Hence,

$$
\operatorname{DSS}\left(T^{1++}(G)\right)=\left[\begin{array}{cc}
2(n-1+r)^{2}\left(J_{n}-I_{n}\right) & \left((n-1+r)^{2}+r^{2}\right) J_{n \times m} \\
\left(r^{2}+(n-1+r)^{2}\right) J_{m \times n} & 2 r^{2}\left(J_{m}-I_{m}\right)
\end{array}\right]
$$

Therefore,

$$
\begin{aligned}
P_{D S S\left(T^{1++}(G)\right)}(\mu) & =\left|\mu I-D S S\left(T^{1++}(G)\right)\right| \\
& =\left|\begin{array}{cc}
\left(\mu+2(n-1+r)^{2}\right) I_{n}-2(n-1+r)^{2} J_{n} & -\left((n-1+r)^{2}+r^{2}\right) J_{n \times m} \\
-\left((n-1+r)^{2}+r^{2}\right) J_{m \times n} & \left(\mu+2 r^{2}\right) I_{m}-2 r^{2} J_{m}
\end{array}\right| .
\end{aligned}
$$


InternationalJournalofAppliedEngineeringResearch,ISSN0973-4562Volume13,Number19(2018)pp.14060-14078 ResearchIndiaPublications, https://dx.doi.org/10.37622/IJAER/13.19.2018.14060-14078

Now using Lemma 1, we get the desired result.

Theorem 31. If $G$ is an $r$-regular graph of order $n$ and size $m$, then

$$
\begin{aligned}
P_{D S S\left(T^{-++}(G)\right)}(\mu)= & {\left[\mu+2(n-1)^{2}\right]^{n-1}\left[\mu+2 r^{2}\right]^{m-1}\left(\left[\mu-2(n-1)^{3}\right]\left[\mu-2(m-1) r^{2}\right]\right.} \\
& \left.-m n\left((n-1)^{2}+r^{2}\right)^{2}\right) .
\end{aligned}
$$

Proof. The graph $T^{-++}(G)$ of an $r$-regular graph $G$ has two types of vertices. From Propositions 23 and 24, the $n$ vertices have degree $n-1$ and $m$ vertices have degree $r$. Hence,

$$
\operatorname{DSS}\left(T^{-++}(G)\right)=\left[\begin{array}{cc}
2(n-1)^{2}\left(J_{n}-I_{n}\right) & \left((n-1)^{2}+r^{2}\right) J_{n \times m} \\
\left(r^{2}+(n-1)^{2}\right) J_{m \times n} & 2 r^{2}\left(J_{m}-I_{m}\right)
\end{array}\right]
$$

Therefore,

$$
\begin{aligned}
P_{D S S\left(T^{-++}(G)\right)}(\mu) & =\left|\mu I-\operatorname{DSS}\left(T^{-++}(G)\right)\right| \\
& =\left|\begin{array}{cc}
\left(\mu+2(n-1)^{2}\right) I_{n}-2(n-1)^{2} J_{n} & -\left((n-1)^{2}+r^{2}\right) J_{n \times m} \\
-\left((n-1)^{2}+r^{2}\right) J_{m \times n} & \left(\mu+2 r^{2}\right) I_{m}-2 r^{2} J_{m}
\end{array}\right| .
\end{aligned}
$$

Now using Lemma 1, we get the desired result.

Theorem 32. If $G$ is an $r$-regular graph of order $n$ and size $m$, then

$$
\begin{aligned}
P_{D S S\left(T^{0-+}(G)\right)}(\mu)= & {\left[\mu+2 r^{2}\right]^{n-1}\left[\mu+(m-2 r+3)^{2}\right]^{m-1}\left(\left[\mu-2(n-1) r^{2}\right]\left[\mu-2(m-1)(m-2 r+3)^{2}\right]\right.} \\
& \left.-m n\left(r^{2}+(m-2 r+3)^{2}\right)^{2}\right) .
\end{aligned}
$$

Proof. The graph $T^{0-+}(G)$ of an $r$-regular graph $G$ has two types of vertices. From Propositions 23 and 24, the $n$ vertices have degree $r$ and $m$ vertices have degree $m-2 r+3$. Hence,

$$
D S S\left(T^{0-+}(G)\right)=\left[\begin{array}{cc}
2 r^{2}\left(J_{n}-I_{n}\right) & \left(r^{2}+(m-2 r+3)^{2}\right) J_{n \times m} \\
\left(r^{2}+(m-2 r+3)^{2}\right) J_{m \times n} & 2(m-2 r+3)^{2}\left(J_{m}-I_{m}\right)
\end{array}\right]
$$

Therefore,

$$
\begin{aligned}
P_{D S S\left(T^{0-+}(G)\right)}(\mu) & =\left|\mu I-\operatorname{DSS}\left(T^{0-+}(G)\right)\right| \\
& =\left|\begin{array}{cc}
\left(\mu+2 r^{2}\right) I_{n}-2 r^{2} J_{n} & -\left(r^{2}+(m-2 r+3)^{2}\right) J_{n \times m} \\
-\left(r^{2}+(m-2 r+3)^{2}\right) J_{m \times n} & \left(\mu+2(m-2 r+3)^{2}\right) I_{m}-2(m-2 r+3)^{2} J_{m}
\end{array}\right| .
\end{aligned}
$$

Now using Lemma 1, we get the desired result.

Theorem 33. If $G$ is an $r$-regular graph of order $n$ and size $m$, then

$$
\begin{aligned}
P_{D S S\left(T^{1-+}(G)\right)}(\mu)= & {\left[\mu+2(n-1+r)^{2}\right]^{n-1}\left[\mu+(m-2 r+3)^{2}\right]^{m-1}\left(\left[\mu-2(n-1)(n-1+r)^{2}\right][\mu\right.} \\
& \left.\left.-2(m-1)(m-2 r+3)^{2}\right]-m n\left((n-1+r)^{2}+(m-2 r+3)^{2}\right)^{2}\right) .
\end{aligned}
$$

Proof. The graph $T^{1-+}(G)$ of an $r$-regular graph $G$ has two types of vertices. From Propositions 23 and 24, the $n$ vertices have degree $n-1+r$ and $m$ vertices have degree $m-2 r+3$. Hence,

$$
\operatorname{DSS}\left(T^{1-+}(G)\right)=\left[\begin{array}{cc}
(n-1+r)^{2}\left(J_{n}-I_{n}\right) & \left((n-1+r)^{2}+(m-2 r+3)^{2}\right) J_{n \times m} \\
\left((n-1+r)^{2}+(m-2 r+3)^{2}\right) J_{m \times n} & 2(m-2 r+3)^{2}\left(J_{m}-I_{m}\right)
\end{array}\right]
$$

Therefore,

$$
\begin{aligned}
P_{D S S\left(T^{1-+}(G)\right)}(\mu) & =\left|\mu I-D S S\left(T^{1-+}(G)\right)\right| \\
& =\left|\begin{array}{cc}
\left(\mu+2(n-1+r)^{2}\right) I_{n}-2(n-1+r)^{2} J_{n} & -\left((n-1+r)^{2}+(m-2 r+3)^{2}\right) J_{n \times m} \\
-\left((n-1+r)^{2}+(m-2 r+3)^{2}\right) J_{m \times n} & \left(\mu+2(m-2 r+3)^{2}\right) I_{m}-2(m-2 r+3)^{2} J_{m}
\end{array}\right| .
\end{aligned}
$$


InternationalJournalofAppliedEngineeringResearch,ISSN0973-4562Volume13,Number19(2018)pp.14060-14078

ResearchIndiaPublications, https://dx.doi.org/10.37622/IJAER/13.19.2018.14060-14078

Now using Lemma 1, we get the desired result.

Theorem 34. If $G$ is an $r$-regular graph of order $n$ and size $m$, then

$$
\begin{aligned}
P_{D S S\left(T^{+-+}(G)\right)}(\mu)= & {\left[\mu+8 r^{2}\right]^{n-1}\left[\mu+(m-2 r+3)^{2}\right]^{m-1}\left(\left[\mu-8(n-1) r^{2}\right]\left[\mu-2(m-1)(m-2 r+3)^{2}\right]\right.} \\
& \left.-m n\left(4 r^{2}+(m-2 r+3)^{2}\right)^{2}\right) .
\end{aligned}
$$

Proof. The graph $T^{+-+}(G)$ of an $r$-regular graph $G$ has two types of vertices. From Propositions 23 and 24, the $n$ vertices have degree $2 r$ and $m$ vertices have degree $m-2 r+3$. Hence,

$$
\operatorname{DSS}\left(T^{+-+}(G)\right)=\left[\begin{array}{cc}
8 r^{2}\left(J_{n}-I_{n}\right) & \left(4 r^{2}+(m-2 r+3)^{2}\right) J_{n \times m} \\
\left(4 r^{2}+(m-2 r+3)^{2}\right) J_{m \times n} & 2(m-2 r+3)^{2}\left(J_{m}-I_{m}\right)
\end{array}\right]
$$

Therefore,

$$
\begin{aligned}
P_{D S S\left(T^{+-+}(G)\right)}(\mu) & =\left|\mu I-D S S\left(T^{+-+}(G)\right)\right| \\
& =\left|\begin{array}{cc}
\left(\mu+8 r^{2}\right) I_{n}-8 r^{2} J_{n} & -\left(4 r^{2}+(m-2 r+3)^{2}\right) J_{n \times m} \\
-\left(4 r^{2}+(m-2 r+3)^{2}\right) J_{m \times n} & \left(\mu+2(m-2 r+3)^{2}\right) I_{m}-2(m-2 r+3)^{2} J_{m}
\end{array}\right| .
\end{aligned}
$$

Now using Lemma 1, we get the desired result.

Theorem 35. If $G$ is an $r$-regular graph of order $n$ and size $m$, then

$$
\begin{aligned}
P_{D S S\left(T^{--+}(G)\right)}(\mu)= & {\left[\mu+2(n-1)^{2}\right]^{n-1}\left[\mu+(m-2 r+3)^{2}\right]^{m-1}\left(\left[\mu-2(n-1)^{3}\right][\mu\right.} \\
& \left.\left.-2(m-1)(m-2 r+3)^{2}\right]-m n\left((n-1)^{2}+(m-2 r+3)^{2}\right)^{2}\right) .
\end{aligned}
$$

Proof. The graph $T^{--+}(G)$ of an $r$-regular graph $G$ has two types of vertices. From Propositions 23 and 24, the $n$ vertices have degree $n-1$ and $m$ vertices have degree $m-2 r+3$. Hence,

$$
\operatorname{DSS}\left(T^{--+}(G)\right)=\left[\begin{array}{cc}
2(n-1)^{2}\left(J_{n}-I_{n}\right) & \left((n-1)^{2}+(m-2 r+3)^{2}\right) J_{n \times m} \\
\left((n-1)^{2}+(m-2 r+3)^{2}\right) J_{m \times n} & 2(m-2 r+3)^{2}\left(J_{m}-I_{m}\right)
\end{array}\right]
$$

Therefore,

$$
\begin{aligned}
P_{D S S\left(T^{--+}(G)\right)}(\mu) & =\left|\mu I-\operatorname{DSS}\left(T^{--+}(G)\right)\right| \\
& =\left|\begin{array}{cc}
\left(\mu+2(n-1)^{2}\right) I_{n}-2(n-1)^{2} J_{n} & -\left((n-1)^{2}+(m-2 r+3)^{2}\right) J_{n \times m} \\
-\left((n-1)^{2}+(m-2 r+3)^{2}\right) J_{m \times n} & \left(\mu+2(m-2 r+3)^{2}\right) I_{m}-2(m-2 r+3)^{2} J_{m}
\end{array}\right| .
\end{aligned}
$$

Now using Lemma 1, we get the desired result.

The degree square sum polynomials of the transformation graphs $T^{00+}(G)$ (subdivision graph $S(G)$ ), $T^{+0+}(G)$ (semitotal point graph $\left.T_{2}(G)\right), T^{0++}(G)$ (semitotal line graph $T_{1}(G)$ ), $T^{+++}(G)$ (total graph $T(G)$ ) are already computed in [4].

Theorem 36. If $G$ is an $r$-regular graph of order $n$ and size $m$, then

$$
\begin{aligned}
P_{D S S\left(T^{00-}(G)\right)}(\mu)= & {\left[\mu+2(m-r)^{2}\right]^{n-1}\left[\mu+2(n-2)^{2}\right]^{m-1}\left(\left[\mu-2(n-1)(m-r)^{2}\right][\mu\right.} \\
& \left.\left.-2(m-1)(n-2)^{2}\right]-m n\left((m-r)^{2}+(n-2)^{2}\right)^{2}\right) .
\end{aligned}
$$

Proof. The graph $T^{00-}(G)$ of an $r$-regular graph $G$ has two types of vertices. From Propositions 23 and 24, the $n$ vertices have degree $m-r$ and $m$ vertices have degree $n-2$. Hence,

$$
\operatorname{DSS}\left(T^{00-}(G)\right)=\left[\begin{array}{cc}
2(m-r)^{2}\left(J_{n}-I_{n}\right) & \left((m-r)^{2}+(n-2)^{2}\right) J_{n \times m} \\
\left((m-r)^{2}+(n-2)^{2}\right) J_{m \times n} & 2(n-2)^{2}\left(J_{m}-I_{m}\right)
\end{array}\right]
$$


InternationalJournalofAppliedEngineeringResearch,ISSN0973-4562Volume13,Number19(2018)pp.14060-14078 ResearchIndiaPublications, https://dx.doi.org/10.37622/IJAER/13.19.2018.14060-14078

Therefore,

$$
\begin{aligned}
P_{D S S\left(T^{00-}(G)\right)}(\mu) & =\left|\mu I-D S S\left(T^{00-}(G)\right)\right| \\
& =\left|\begin{array}{cc}
\left(\mu+2(m-r)^{2}\right) I_{n}-2(m-r)^{2} J_{n} & -\left((m-r)^{2}+(n-2)^{2}\right) J_{n \times m} \\
-\left((m-r)^{2}+(n-2)^{2}\right) J_{m \times n} & \left(\mu+2(n-2)^{2}\right) I_{m}-2(n-2)^{2} J_{m}
\end{array}\right| .
\end{aligned}
$$

Now using Lemma 1, we get the desired result.

Theorem 37. If $G$ is an $r$-regular graph of order $n$ and size $m$, then

$$
\begin{aligned}
P_{D S S\left(T^{10-}(G)\right)}(\mu)= & {\left[\mu+2(n+m-1-r)^{2}\right]^{n-1}\left[\mu+2(n-2)^{2}\right]^{m-1}\left(\left[\mu-2(n-1)(n+m-1-r)^{2}\right][\mu\right.} \\
& \left.\left.-2(m-1)(n-2)^{2}\right]-m n\left((n+m-1-r)^{2}+(n-2)^{2}\right)^{2}\right) .
\end{aligned}
$$

Proof. The graph $T^{10-}(G)$ of an $r$-regular graph $G$ has two types of vertices. From Propositions 23 and 24, the $n$ vertices have degree $n+m-1-r$ and $m$ vertices have degree $n-2$. Hence,

$$
\operatorname{DSS}\left(T^{10-}(G)\right)=\left[\begin{array}{cc}
2(n+m-1-r)^{2}\left(J_{n}-I_{n}\right) & \left((n+m-1-r)^{2}+(n-2)^{2}\right) J_{n \times m} \\
\left((n+m-1-r)^{2}+(n-2)^{2}\right) J_{m \times n} & 2(n-2)^{2}\left(J_{m}-I_{m}\right)
\end{array}\right]
$$

Therefore,

$$
\begin{aligned}
P_{D S S\left(T^{10-}(G)\right)}(\mu) & =\left|\mu I-D S S\left(T^{10-}(G)\right)\right| \\
& =\left|\begin{array}{cc}
\left(\mu+2(n+m-1-r)^{2}\right) I_{n}-2(n+m-1-r)^{2} J_{n} & -\left((n+m-1-r)^{2}+(n-2)^{2}\right) J_{n \times m} \\
-\left((n+m-1-r)^{2}+(n-2)^{2}\right) J_{m \times n} & \left(\mu+2(n-2)^{2}\right) I_{m}-2(n-2)^{2} J_{m}
\end{array}\right| .
\end{aligned}
$$

Now using Lemma 1, we get the desired result.

Theorem 38. If $G$ is an $r$-regular graph of order $n$ and size $m$, then

$$
\begin{aligned}
P_{D S S\left(T^{+0-}(G)\right)}(\mu)= & {\left[\mu+2 m^{2}\right]^{n-1}\left[\mu+2(n-2)^{2}\right]^{m-1}\left(\left[\mu-2(n-1) m^{2}\right]\left[\mu-2(m-1)(n-2)^{2}\right]\right.} \\
& \left.-m n\left(m^{2}+(n-2)^{2}\right)^{2}\right) .
\end{aligned}
$$

Proof. The graph $T^{+0-}(G)$ of an $r$-regular graph $G$ has two types of vertices. From Propositions 23 and 24, the $n$ vertices have degree $m$ and $m$ vertices have degree $n-2$. Hence,

$$
\operatorname{DSS}\left(T^{+0-}(G)\right)=\left[\begin{array}{cc}
2 m^{2}\left(J_{n}-I_{n}\right) & \left(m^{2}+(n-2)^{2}\right) J_{n \times m} \\
\left(m^{2}+(n-2)^{2}\right) J_{m \times n} & 2(n-2)^{2}\left(J_{m}-I_{m}\right)
\end{array}\right]
$$

Therefore,

$$
\begin{aligned}
P_{D S S\left(T^{+0-}(G)\right)}(\mu) & =\left|\mu I-\operatorname{DSS}\left(T^{+0-}(G)\right)\right| \\
& =\left|\begin{array}{cc}
\left(\mu+2 m^{2}\right) I_{n}-2 m^{2} J_{n} & -\left(m^{2}+(n-2)^{2}\right) J_{n \times m} \\
-\left(m^{2}+(n-2)^{2}\right) J_{m \times n} & \left(\mu+2(n-2)^{2}\right) I_{m}-2(n-2)^{2} J_{m}
\end{array}\right| .
\end{aligned}
$$

Now using Lemma 1, we get the desired result.

Theorem 39. If $G$ is an $r$-regular graph of order $n$ and size $m$, then

$$
\begin{aligned}
P_{D S S\left(T^{-0-}(G)\right)}(\mu)= & {\left[\mu+2(n+m-1-2 r)^{2}\right]^{n-1}\left[\mu+2(n-2)^{2}\right]^{m-1}([\mu} \\
& \left.-2(n-1)(n+m-1-2 r)^{2}\right]\left[\mu-2(m-1)(n-2)^{2}\right] \\
& \left.-m n\left((n+m-1-2 r)^{2}+(n-2)^{2}\right)^{2}\right) .
\end{aligned}
$$


InternationalJournalofAppliedEngineeringResearch,ISSN0973-4562Volume13,Number19(2018)pp.14060-14078

ResearchIndiaPublications, https://dx.doi.org/10.37622/IJAER/13.19.2018.14060-14078

Proof. The graph $T^{-0-}(G)$ of an $r$-regular graph $G$ has two types of vertices. From Propositions 23 and 24 , the $n$ vertices have degree $n+m-1-2 r$ and $m$ vertices have degree $n-2$. Hence,

$$
\operatorname{DSS}\left(T^{-0-}(G)\right)=\left[\begin{array}{cc}
2(n+m-1-2 r)^{2}\left(J_{n}-I_{n}\right) & \left((n+m-1-2 r)^{2}+(n-2)^{2}\right) J_{n \times m} \\
\left((n+m-1-2 r)^{2}+(n-2)^{2}\right) J_{m \times n} & 2(n-2)^{2}\left(J_{m}-I_{m}\right)
\end{array}\right]
$$

Therefore,

$$
\begin{aligned}
P_{D S S\left(T^{-0-}(G)\right)}(\mu) & =\left|\mu I-D S S\left(T^{-0-}(G)\right)\right| \\
& =\left|\begin{array}{cc}
\left(\mu+2(n+m-1-2 r)^{2}\right) I_{n}-2(n+m-1-2 r)^{2} J_{n} & -\left((n+m-1-2 r)^{2}+(n-2)^{2}\right) J_{n \times m} \\
-\left((n+m-1-2 r)^{2}+(n-2)^{2}\right) J_{m \times n} & \left(\mu+2(n-2)^{2}\right) I_{m}-2(n-2)^{2} J_{m}
\end{array}\right| .
\end{aligned}
$$

Now using Lemma 1, we get the desired result.

Theorem 40. If $G$ is an $r$-regular graph of order $n$ and size $m$, then

$$
\begin{aligned}
P_{D S S\left(T^{01-}(G)\right)}(\mu)= & {\left[\mu+2(m-r)^{2}\right]^{n-1}\left[\mu+2(n+m-3)^{2}\right]^{m-1}\left(\left[\mu-2(n-1)(m-r)^{2}\right][\mu\right.} \\
& \left.\left.-2(m-1)(n+m-3)^{2}\right]-m n\left((m-r)^{2}+(n+m-3)^{2}\right)^{2}\right) .
\end{aligned}
$$

Proof. The graph $T^{01-}(G)$ of an $r$-regular graph $G$ has two types of vertices. From Propositions 23 and 24, the $n$ vertices have degree $m-r$ and $m$ vertices have degree $n+m-3$. Hence,

$$
\operatorname{DSS}\left(T^{01-}(G)\right)=\left[\begin{array}{cc}
2(m-r)^{2}\left(J_{n}-I_{n}\right) & \left((m-r)^{2}+(n+m-3)^{2}\right) J_{n \times m} \\
\left((m-r)^{2}+(n+m-3)^{2}\right) J_{m \times n} & 2(n+m-3)^{2}\left(J_{m}-I_{m}\right)
\end{array}\right]
$$

Therefore,

$$
\begin{aligned}
P_{D S S\left(T^{01-}(G)\right)}(\mu) & =\left|\mu I-D S S\left(T^{01-}(G)\right)\right| \\
& =\left|\begin{array}{cc}
\left(\mu+2(m-r)^{2}\right) I_{n}-2(m-r)^{2} J_{n} & -\left((m-r)^{2}+(n+m-3)^{2}\right) J_{n \times m} \\
-\left((m-r)^{2}+(n+m-3)^{2}\right) J_{m \times n} & \left(\mu+2(n+m-3)^{2}\right) I_{m}-2(n+m-3)^{2} J_{m}
\end{array}\right| .
\end{aligned}
$$

Now using Lemma 1, we get the desired result.

Theorem 41. If $G$ is an $r$-regular graph of order $n$ and size $m$, then

$$
\begin{aligned}
P_{D S S\left(T^{11-}(G)\right)}(\mu)= & {\left[\mu+2(n+m-1-r)^{2}\right]^{n-1}\left[\mu+2(n+m-3)^{2}\right]^{m-1}([\mu} \\
& \left.-2(n-1)(n+m-1-r)^{2}\right]\left[\mu-2(m-1)(n+m-3)^{2}\right] \\
& \left.-m n\left((n+m-1-r)^{2}+(n+m-3)^{2}\right)^{2}\right) .
\end{aligned}
$$

Proof. The graph $T^{11-}(G)$ of an $r$-regular graph $G$ has two types of vertices. From Propositions 23 and 24, the $n$ vertices have degree $n+m-1-r$ and $m$ vertices have degree $n+m-3$. Hence,

$$
\operatorname{DSS}\left(T^{11-}(G)\right)=\left[\begin{array}{cc}
2(n+m-1-r)^{2}\left(J_{n}-I_{n}\right) & \left((n+m-1-r)^{2}+(n+m-3)^{2}\right) J_{n \times m} \\
\left((n+m-1-r)^{2}+(n+m-3)^{2}\right) J_{m \times n} & 2(n+m-3)^{2}\left(J_{m}-I_{m}\right)
\end{array}\right]
$$

Therefore,

$$
\begin{aligned}
P_{D S S\left(T^{11-}(G)\right)}(\mu) & =\left|\mu I-D S S\left(T^{11-}(G)\right)\right| \\
& =\left|\begin{array}{cc}
\left(\mu+2(n+m-1-r)^{2}\right) I_{n}-2(n+m-1-r)^{2} J_{n} & -\left((n+m-1-r)^{2}+(n+m-3)^{2}\right) J_{n \times m} \\
-\left((n+m-1-r)^{2}+(n+m-3)^{2}\right) J_{m \times n} & \left(\mu+2(n+m-3)^{2}\right) I_{m}-2(n+m-3)^{2} J_{m}
\end{array}\right| .
\end{aligned}
$$

Now using Lemma 1, we get the desired result. 
InternationalJournalofAppliedEngineeringResearch,ISSN0973-4562Volume13,Number19(2018)pp.14060-14078

ResearchIndiaPublications, https://dx.doi.org/10.37622/IJAER/13.19.2018.14060-14078

Theorem 42. If $G$ is an $r$-regular graph of order $n$ and size $m$, then

$$
\begin{aligned}
P_{D S S\left(T^{+1-}(G)\right)}(\mu)= & {\left[\mu+2 m^{2}\right]^{n-1}\left[\mu+2(n+m-3)^{2}\right]^{m-1}\left(\left[\mu-2(n-1) m^{2}\right][\mu\right.} \\
& \left.\left.-2(m-1)(n+m-3)^{2}\right]-m n\left(m^{2}+(n+m-3)^{2}\right)^{2}\right) .
\end{aligned}
$$

Proof. The graph $T^{+1-}(G)$ of an $r$-regular graph $G$ has two types of vertices. From Propositions 23 and 24, the $n$ vertices have degree $m$ and $m$ vertices have degree $n+m-3$. Hence,

$$
\operatorname{DSS}\left(T^{+1-}(G)\right)=\left[\begin{array}{cc}
2 m^{2}\left(J_{n}-I_{n}\right) & \left(m^{2}+(n+m-3)^{2}\right) J_{n \times m} \\
\left(m^{2}+(n+m-3)^{2}\right) J_{m \times n} & 2(n+m-3)^{2}\left(J_{m}-I_{m}\right)
\end{array}\right]
$$

Therefore,

$$
\begin{aligned}
P_{D S S\left(T^{+1-}(G)\right)}(\mu) & =\left|\mu I-\operatorname{DSS}\left(T^{+1-}(G)\right)\right| \\
& =\left|\begin{array}{cc}
\left(\mu+2 m^{2}\right) I_{n}-2 m^{2} J_{n} & -\left(m^{2}+(n+m-3)^{2}\right) J_{n \times m} \\
-\left(m^{2}+(n+m-3)^{2}\right) J_{m \times n} & \left(\mu+2(n+m-3)^{2}\right) I_{m}-2(n+m-3)^{2} J_{m}
\end{array}\right| .
\end{aligned}
$$

Now using Lemma 1, we get the desired result.

Theorem 43. If $G$ is an $r$-regular graph of order $n$ and size $m$, then

$$
P_{D S S\left(T^{-1-}(G)\right)}(\mu)=\left[\mu+2 R_{1}^{2}\right]^{n-1}\left[\mu+2 R_{2}^{2}\right]^{m-1}\left(\left[\mu-2(n-1) R_{1}^{2}\right]\left[\mu-2(m-1) R_{2}^{2}\right]-m n\left(R_{1}^{2}+R_{2}^{2}\right)^{2}\right) .
$$

Proof. The graph $T^{-1-}(G)$ of an $r$-regular graph $G$ has two types of vertices. From Propositions 23 and 24, the $n$ vertices have degree $R_{1}=n+m-1-2 r$ and $m$ vertices have degree $R_{2}=n+m-3$. Hence,

$$
\operatorname{DSS}\left(T^{-1-}(G)\right)=\left[\begin{array}{cc}
2 R_{1}^{2}\left(J_{n}-I_{n}\right) & \left(R_{1}^{2}+R_{2}^{2}\right) J_{n \times m} \\
\left(R_{1}^{2}+R_{2}^{2}\right) J_{m \times n} & 2 R_{2}^{2}\left(J_{m}-I_{m}\right)
\end{array}\right]
$$

Therefore,

$$
\begin{aligned}
P_{D S S\left(T^{-1-}(G)\right)}(\mu) & =\left|\mu I-\operatorname{DSS}\left(T^{-1-}(G)\right)\right| \\
& =\left|\begin{array}{cc}
\left(\mu+2 R_{1}^{2}\right) I_{n}-2 R_{1}^{2} J_{n} & -\left(R_{1}^{2}+R_{2}^{2}\right) J_{n \times m} \\
-\left(R_{1}^{2}+R_{2}^{2}\right) J_{m \times n} & \left(\mu+2 R_{2}^{2}\right) I_{m}-2 R_{2}^{2} J_{m}
\end{array}\right| .
\end{aligned}
$$

Now using Lemma 1, we get the desired result.

Theorem 44. If $G$ is an $r$-regular graph of order $n$ and size $m$, then

$$
\begin{aligned}
P_{D S S\left(T^{0+-}(G)\right)}(\mu)= & {\left[\mu+2(m-r)^{2}\right]^{n-1}\left[\mu+2(n+2(r-2))^{2}\right]^{m-1}\left(\left[\mu-2(n-1)(m-r)^{2}\right][\mu\right.} \\
& \left.\left.-2(m-1)(n+2(r-2))^{2}\right]-m n\left((m-r)^{2}+(n+2(r-2))^{2}\right)^{2}\right) .
\end{aligned}
$$

Proof. The graph $T^{0+-}(G)$ of an $r$-regular graph $G$ has two types of vertices. From Propositions 23 and 24, the $n$ vertices have degree $m-r$ and $m$ vertices have degree $n+2(r-2)$. Hence,

$$
\operatorname{DSS}\left(T^{0+-}(G)\right)=\left[\begin{array}{cc}
2(m-r)^{2}\left(J_{n}-I_{n}\right) & \left((m-r)^{2}+(n+2(r-2))^{2}\right) J_{n \times m} \\
\left((m-r)^{2}+(n+2(r-2))^{2}\right) J_{m \times n} & 2(n+2(r-2))^{2}\left(J_{m}-I_{m}\right)
\end{array}\right]
$$

Therefore,

$$
\begin{aligned}
P_{D S S\left(T^{0+-}(G)\right)}(\mu) & =\left|\mu I-\operatorname{DSS}\left(T^{0+-}(G)\right)\right| \\
& =\left|\begin{array}{cc}
\left(\mu+2(m-r)^{2}\right) I_{n}-2(m-r)^{2} J_{n} & -\left((m-r)^{2}+(n+2(r-2))^{2}\right) J_{n \times m} \\
-\left((m-r)^{2}+(n+2(r-2))^{2}\right) J_{m \times n} & \left(\mu+2(n+2(r-2))^{2}\right) I_{m}-2(n+2(r-2))^{2} J_{m}
\end{array}\right| .
\end{aligned}
$$


InternationalJournalofAppliedEngineeringResearch,ISSN0973-4562Volume13,Number19(2018)pp.14060-14078 ResearchIndiaPublications, https://dx.doi.org/10.37622/IJAER/13.19.2018.14060-14078

Now using Lemma 1, we get the desired result.

Theorem 45. If $G$ is an $r$-regular graph of order $n$ and size $m$, then

$$
P_{D S S\left(T^{1+-}(G)\right)}(\mu)=\left[\mu+2 R_{3}^{2}\right]^{n-1}\left[\mu+2 R_{4}^{2}\right]^{m-1}\left(\left[\mu-2(n-1) R_{3}^{2}\right]\left[\mu-2(m-1) R_{4}^{2}\right]-m n\left(R_{3}^{2}+R_{4}^{2}\right)^{2}\right) .
$$

Proof. The graph $T^{1+-}(G)$ of an $r$-regular graph $G$ has two types of vertices. From Propositions 23 and 24, the $n$ vertices have degree $R_{3}=n+m-1-r$ and $m$ vertices have degree $R_{4}=n+2(r-2)$. Hence,

$$
\operatorname{DSS}\left(T^{1+-}(G)\right)=\left[\begin{array}{cc}
2 R_{3}^{2}\left(J_{n}-I_{n}\right) & \left(R_{3}^{2}+R_{4}^{2}\right) J_{n \times m} \\
\left(R_{3}^{2}+R_{4}^{2}\right) J_{m \times n} & 2 R_{4}^{2}\left(J_{m}-I_{m}\right)
\end{array}\right]
$$

Therefore,

$$
\begin{aligned}
P_{D S S\left(T^{1+-}(G)\right)}(\mu) & =\left|\mu I-\operatorname{DSS}\left(T^{1+-}(G)\right)\right| \\
& =\left|\begin{array}{cc}
\left(\mu+2 R_{3}^{2}\right) I_{n}-2 R_{3}^{2} J_{n} & -\left(R_{3}^{2}+R_{4}^{2}\right) J_{n \times m} \\
-\left(R_{3}^{2}+R_{4}^{2}\right) J_{m \times n} & \left(\mu+2 R_{4}^{2}\right) I_{m}-2 R_{4}^{2} J_{m}
\end{array}\right| .
\end{aligned}
$$

Now using Lemma 1, we get the desired result.

Theorem 46. If $G$ is an $r$-regular graph of order $n$ and size $m$, then

$$
\begin{aligned}
P_{D S S\left(T^{++-}(G)\right)}(\mu)= & {\left[\mu+2 m^{2}\right]^{n-1}\left[\mu+2(n+2(r-2))^{2}\right]^{m-1}\left(\left[\mu-2(n-1) m^{2}\right][\mu\right.} \\
& \left.\left.-2(m-1)(n+2(r-2))^{2}\right]-m n\left(m^{2}+(n+2(r-2))^{2}\right)^{2}\right) .
\end{aligned}
$$

Proof. The graph $T^{++-}(G)$ of an $r$-regular graph $G$ has two types of vertices. From Propositions 23 and 24, the $n$ vertices have degree $m$ and $m$ vertices have degree $n+2(r-2)$. Hence,

$$
\operatorname{DSS}\left(T^{++-}(G)\right)=\left[\begin{array}{cc}
2 m^{2}\left(J_{n}-I_{n}\right) & \left(m^{2}+(n+2(r-2))^{2}\right) J_{n \times m} \\
\left(m^{2}+(n+2(r-2))^{2}\right) J_{m \times n} & 2(n+2(r-2))^{2}\left(J_{m}-I_{m}\right)
\end{array}\right]
$$

Therefore,

$$
\begin{aligned}
P_{D S S\left(T^{++-}(G)\right)}(\mu) & =\left|\mu I-\operatorname{DSS}\left(T^{++-}(G)\right)\right| \\
& =\left|\begin{array}{cc}
\left(\mu+2 m^{2}\right) I_{n}-2 m^{2} J_{n} & -\left(m^{2}+(n+2(r-2))^{2}\right) J_{n \times m} \\
-\left(m^{2}+(n+2(r-2))^{2}\right) J_{m \times n} & \left(\mu+2(n+2(r-2))^{2}\right) I_{m}-2(n+2(r-2))^{2} J_{m}
\end{array}\right| .
\end{aligned}
$$

Now using Lemma 1, we get the desired result.

Theorem 47. If $G$ is an $r$-regular graph of order $n$ and size $m$, then

$$
\begin{aligned}
P_{D S S\left(T^{-+-}(G)\right)}(\mu)= & {\left[\mu+2 R_{5}^{2}\right]^{n-1}\left[\mu+2 R_{6}^{2}\right]^{m-1}\left(\left[\mu-2(n-1) R_{5}^{2}\right]\left[\mu-2(m-1) R_{6}^{2}\right]\right.} \\
& \left.-m n\left(R_{5}^{2}+R_{6}^{2}\right)^{2}\right) .
\end{aligned}
$$

Proof. The graph $T^{-+-}(G)$ of an $r$-regular graph $G$ has two types of vertices. From Propositions 23 and 24, the $n$ vertices have degree $R_{5}=n+m-1-2 r$ and $m$ vertices have degree $R_{6}=n+2(r-2)$. Hence,

$$
\operatorname{DSS}\left(T^{-+-}(G)\right)=\left[\begin{array}{cc}
2 R_{5}^{2}\left(J_{n}-I_{n}\right) & \left(R_{5}^{2}+R_{6}^{2}\right) J_{n \times m} \\
\left(R_{5}^{2}+R_{6}^{2}\right) J_{m \times n} & 2 R_{6}^{2}\left(J_{m}-I_{m}\right)
\end{array}\right]
$$

Therefore,

$$
\begin{aligned}
P_{D S S\left(T^{-+-}(G)\right)}(\mu) & =\left|\mu I-\operatorname{DSS}\left(T^{-+-}(G)\right)\right| \\
& =\left|\begin{array}{cc}
\left(\mu+2 R_{5}^{2}\right) I_{n}-2 R_{5}^{2} J_{n} & -\left(R_{5}^{2}+R_{6}^{2}\right) J_{n \times m} \\
-\left(R_{5}^{2}+R_{6}^{2}\right) J_{m \times n} & \left(\mu+2 R_{6}^{2}\right) I_{m}-2 R_{6}^{2} J_{m}
\end{array}\right| .
\end{aligned}
$$


InternationalJournalofAppliedEngineeringResearch,ISSN0973-4562Volume13,Number19(2018)pp.14060-14078

ResearchIndiaPublications, https://dx.doi.org/10.37622/IJAER/13.19.2018.14060-14078

Now using Lemma 1, we get the desired result.

Theorem 48. If $G$ is an $r$-regular graph of order $n$ and size $m$, then

$$
\begin{aligned}
P_{D S S\left(T^{0--}(G)\right)}(\mu)= & {\left[\mu+2(m-r)^{2}\right]^{n-1}\left[\mu+2(n+m-2 r-1)^{2}\right]^{m-1}\left(\left[\mu-2(n-1)(m-r)^{2}\right][\mu\right.} \\
& \left.\left.-2(m-1)(n+m-2 r-1)^{2}\right]-m n\left((m-r)^{2}+(n+m-2 r-1)^{2}\right)^{2}\right) .
\end{aligned}
$$

Proof. The graph $T^{0--}(G)$ of an $r$-regular graph $G$ has two types of vertices. From Propositions 23 and 24, the $n$ vertices have degree $m-r$ and $m$ vertices have degree $n+m-2 r-1$. Hence,

$$
\operatorname{DSS}\left(T^{0--}(G)\right)=\left[\begin{array}{cc}
2(m-r)^{2}\left(J_{n}-I_{n}\right) & \left((m-r)^{2}+(n+m-2 r-1)^{2}\right) J_{n \times m} \\
\left((m-r)^{2}+(n+m-2 r-1)^{2}\right) J_{m \times n} & 2(n+m-2 r-1)^{2}\left(J_{m}-I_{m}\right)
\end{array}\right]
$$

Therefore,

$$
\begin{aligned}
P_{D S S\left(T^{0--}(G)\right)}(\mu) & =\left|\mu I-D S S\left(T^{0--}(G)\right)\right| \\
& =\left|\begin{array}{cc}
\left(\mu+2(m-r)^{2}\right) I_{n}-2(m-r)^{2} J_{n} & -\left((m-r)^{2}+(n+m-2 r-1)^{2}\right) J_{n \times m} \\
-\left((m-r)^{2}+(n+m-2 r-1)^{2}\right) J_{m \times n} & \left(\mu+2(n+m-2 r-1)^{2}\right) I_{m}-2(n+m-2 r-1)^{2} J_{m}
\end{array}\right| .
\end{aligned}
$$

Now using Lemma 1, we get the desired result.

Theorem 49. If $G$ is an $r$-regular graph of order $n$ and size $m$, then

$$
\begin{aligned}
P_{D S S\left(T^{1--}(G)\right)}(\mu)= & {\left[\mu+2 R_{7}^{2}\right]^{n-1}\left[\mu+2 R_{8}^{2}\right]^{m-1}\left(\left[\mu-2(n-1) R_{7}^{2}\right]\left[\mu-2(m-1) R_{8}^{2}\right]\right.} \\
& \left.-m n\left(R_{7}^{2}+R_{8}^{2}\right)^{2}\right) .
\end{aligned}
$$

Proof. The graph $T^{1--}(G)$ of an $r$-regular graph $G$ has two types of vertices. From Propositions 23 and 24, the $n$ vertices have degree $R_{7}=n+m-1-r$ and $m$ vertices have degree $R_{8}=n+m-2 r-1$. Hence,

$$
\operatorname{DSS}\left(T^{1--}(G)\right)=\left[\begin{array}{cc}
2 R_{7}^{2}\left(J_{n}-I_{n}\right) & \left(R_{7}^{2}+R_{8}^{2}\right) J_{n \times m} \\
\left(R_{7}^{2}+R_{8}^{2}\right) J_{m \times n} & 2 R_{8}^{2}\left(J_{m}-I_{m}\right)
\end{array}\right]
$$

Therefore,

$$
\begin{aligned}
P_{D S S\left(T^{1--}(G)\right)}(\mu) & =\left|\mu I-\operatorname{DSS}\left(T^{1--}(G)\right)\right| \\
& =\left|\begin{array}{cc}
\left(\mu+2 R_{7}^{2}\right) I_{n}-2 R_{7}^{2} J_{n} & -\left(R_{7}^{2}+R_{8}^{2}\right) J_{n \times m} \\
-\left(R_{7}^{2}+R_{8}^{2}\right) J_{m \times n} & \left(\mu+2 R_{8}^{2}\right) I_{m}-2 R_{8}^{2} J_{m}
\end{array}\right| .
\end{aligned}
$$

Now using Lemma 1, we get the desired result.

Theorem 50. If $G$ is an $r$-regular graph of order $n$ and size $m$, then

$$
\begin{aligned}
P_{D S S\left(T^{+-}(G)\right)}(\mu)= & {\left[\mu+2 m^{2}\right]^{n-1}\left[\mu+2(n+m-2 r-1)^{2}\right]^{m-1}\left(\left[\mu-2(n-1) m^{2}\right][\mu\right.} \\
& \left.\left.-2(m-1)(n+m-2 r-1)^{2}\right]-m n\left(m^{2}+(n+m-2 r-1)^{2}\right)^{2}\right) .
\end{aligned}
$$

Proof. The graph $T^{+--}(G)$ of an $r$-regular graph $G$ has two types of vertices. From Propositions 23 and 24, the $n$ vertices have degree $m$ and $m$ vertices have degree $n+m-2 r-1$. Hence,

$$
\operatorname{DSS}\left(T^{+--}(G)\right)=\left[\begin{array}{cc}
2 m^{2}\left(J_{n}-I_{n}\right) & \left(m^{2}+(n+m-2 r-1)^{2}\right) J_{n \times m} \\
\left(m^{2}+(n+m-2 r-1)^{2}\right) J_{m \times n} & 2(n+m-2 r-1)^{2}\left(J_{m}-I_{m}\right)
\end{array}\right]
$$

Therefore,

$$
\begin{aligned}
P_{D S S\left(T^{+--}(G)\right)}(\mu) & =\left|\mu I-\operatorname{DSS}\left(T^{+--}(G)\right)\right| \\
& =\left|\begin{array}{cc}
\left(\mu+2 m^{2}\right) I_{n}-2(n+m-2 r-1)^{2} J_{n} & -\left(m^{2}+(n+m-2 r-1)^{2}\right) J_{n \times m} \\
-\left(m^{2}+(n+m-2 r-1)^{2}\right) J_{m \times n} & \left(\mu+2(n+m-2 r-1)^{2}\right) I_{m}-2(n+m-2 r-1)^{2} J_{m}
\end{array}\right| .
\end{aligned}
$$


InternationalJournalofAppliedEngineeringResearch,ISSN0973-4562Volume13,Number19(2018)pp.14060-14078 ResearchIndiaPublications, https://dx.doi.org/10.37622/IJAER/13.19.2018.14060-14078

Now using Lemma 1, we get the desired result.

Theorem 51. If $G$ is an $r$-regular graph of order $n$ and size $m$, then

$P_{D S S\left(T^{--}(G)\right)}(\mu)=\left[\mu-2 R_{9}^{2}(n+m-1)\right]\left[\mu+2 R_{9}^{2}\right]^{n+m-1}$.

Proof. All the vertices of a graph $T^{---}(G)$ of an $r$-regular graph $G$ have degree $R_{9}=n+m-2 r-1$ from Propositions 23 and 24. Hence, the result follows from Eq. (1).

Similarly one can compute the degree square sum polynomial of transformation graphs $T^{x y 1}(G)$. The degree square sum polynomial of transformation graphs $T^{x y 0}(G)$ can be omitted since they are disconnected graphs and no chemical compound corresponds to those graphs and hence there is no scope for application.

\section{Acknowledgement}

${ }^{*}$ This work is partially supported by the University Grants Commission (UGC), New Delhi, through UGC-SAP DRSIII for 2016-2021: F.510/3/DRS-III/2016(SAP-I).

${ }^{1}$ This work is supported by the DST INSPIRE Fellowship 2017: No.DST/INSPIRE Fellowship/[IF170465].

\section{REFERENCES}

[1] Aouchiche, M., Hansen, P., 2014, Distance spectra of graphs: A survey, Linear Algebra Appl., 458, pp. 301386.

[2] Baoyindureng, W., Jixiang, M., 2001, Basic properties of Total Transformation Graphs, J. Math. Study, 34 (2), pp. 109-116.

[3] Basavanagoud, B., 2018, Basic properties of generalized xyz-Point-Line transformation graphs, J. Inf. Optim. Sci., 39 (2), pp. 561-580, DOI: 10.1080/02522667.2017.1395147.

[4] Basavanagoud, B., Chitra, E., 2018, Degree square sum energy of graphs, Int. J. Math. Appl., 6 (2-B), pp. 193205.

[5] Basavanagoud, B., Gali, C. S., 2018, Computing first and second Zagreb indices of generalized xyz-PointLine transformation graphs, J. Global Res. Math. Arch., 5 (4), pp. 100-122.

[6] Brouwer, A. E., Haemers, W. H., 2012, Spectra of Graphs, Springer, Berlin.

[7] Cvetković, D., Doob, M., Sachs, H., 1980, Spectra of Graphs-Theory and Applications, Academic Press, New York.
[8] Cvetković, D., Rowlinson, P., Simić, S. K., 2007, Eigenvalue bounds for the signless Laplacian, Publ. Inst. Math.(Beograd), 81, pp. 11-27.

[9] Deng, A., Kelmans, A., Meng, J., 2013, Laplacian Spectra of regular graph transformations, Discrete Appl. Math., 161, pp. 118-133.

[10] Gallian, J. A., 2017, A dynamic survey of graph labeling, Electronic J. Comb..

[11] Harary, F., Graph Theory, 1969, Addison-Wesely, Reading, Mass.

[12] Indulal, G., Vijayakumar, A., 2008, A note on energy of some graphs, MATCH Commun. Math. Comput. Chem., 59, pp. 269-274.

[13] Jog, S. R., Hande, S. P., Revankar, D. S., 2013, Degree sum polynomial of graph valued functions on regular graphs, Int. J. Graph Theory, 1, pp. 108-115.

[14] Kulli, V. R., Biradar, M. S., 2002, The line splitting graph of a graph, Acta Ciencia Indica, 28 (3), pp. 317-322.

[15] Kulli, V. R., 2012, College Graph Theory, Vishwa International Publications, Gulbarga, India.

[16] Mohar, B., 1991, The Laplacian spectrum of graphs, in: Y. Alavi, G. Chartrand, O. R. Ollermann, A. J. Schwenk (Eds.), Graph Theory, Combinatorics and Applications, Wiley, New York, 2, pp. 871-898.

[17] Ponraj, R., Somasundaram, S., 2004, On the degree splitting graph of a graph, Natl. Acad, Sci. Letters., 27 (7 and 8), pp. 275-278.

[18] Ramane, H. S., Gudimani, S. B., Shinde, S. S., 2013, Signless Laplacian polynomial and characteristic polynomial of a graph, J. Discrete Math., Article ID 105624, pp. 4 pages. http://dx.doi.org/10.1155/2013/105624.

[19] Ramane, H. S., Revankar, D. S., Patil, J. B., 2013, Bounds for the degree sum eigenvalues and degree sum energy of a graph, Int. J. Pure Appl. Math. Sci., 6, pp. 161-167.

[20] Ramane, H. S., Shinde, S. S., 2017, Degree exponent polynomial of graphs obtained by some graph operations, Electronic Notes in Discrete Math., 63, pp. 161-168.

[21] Sampathkumar, E., Walikar, H. B., 1980-81, On splitting graph of a graph, J. Karnatak Univ. Sci., 25 and 26 (combined), pp. 13-16. 